\title{
Informing Relatives about Schizophrenia
}

G. M. Carstairs, D. F. Early, H. R. Rollin and J. K. Wing, Medical Advisers to the National Schizophrenia Fellowship (Correspondence: Professor J. K. Wing, Institute of Psychiatry, London SE5)

One of the chief problems mentioned by the relatives of people suffering from schizophrenia is the difficulty in obtaining factual and practical advice. Many have struggled on for years, using trial-and-error methods, without knowing whether their lack of success was due to the immutable course of events, to ignorance, or to a deficiency in themselves of the ability to care. Some have made tentative enquiries but have been given a more or less polite brush-off. Although most relatives, like most sufferers, do eventually become aware of the diagnosis, it is rare for them to be given this information as part of a longterm plan of management which they are invited to share with professional staff and patient, and even more unusual for them to be told early in the course of the disorder.

This would not be true in other branches of medicine. In the case of some long-term disabling diseases such as Parkinson's disease or diabetes, or following colostomy, it is possible to discover specialist clinics within a reasonable distance where patients and relatives can be confident that professional knowledge will be placed at their disposal in an understandable form and the limits of what can be done explained.

Psychiatrists have for some time been using aids to selfmanagement in depressive and anxiety disorders. Similar methods have been used experimentally with families who 'live with schizoprehnia', but this has not become as yet a part of common clinical practice. There are three main reasons why clinicians might be reluctant to include relatives in such a scheme; the first is confidentiality, the second is dislike of 'labelling', the third is unfamiliarity with the principles involved.

\section{Confidentiality}

Some psychiatrists state that they will not discuss a patient's affairs with relatives without specific permission, even when refusal is due to lack of insight into the nature and effects of abnormal beliefs and behaviour. This extreme position is difficult to justify in the light of all the evidence that environmental, particularly family, factors can influence the course of illness for better or worse. It may occasionally be possible to afford the luxury of two counsellors, one advising the relative, the other the patient. but they should be part of the same team and thus be able to give complete advice. More commonly, it is necessary to help the patient, as well as the relatives, to understand that living with schizophrenia is much easier if the problems are shared. Other members of the family ('caring relatives') have a right to know.

\section{Labelling}

A clinician may be in doubt about the diagnosis during the early stages of a disorder, or, understandably, be reluctant to use a term such as 'schizophrenia' if the acute phase could well clear up with no persisting disability. Relatives will usually accept a frank admission of the uncertainties in making a diagnosis-doctors do not need to appear omniscient. It is important to recognize, however, that relatives, as well as patients, are often aware that schizophrenia is a possible diagnosis. Many will have looked up the illness in books readily available in their local library, so that avoiding any mention of it can lead to much greater confusion and inappropriate behaviour towards the patient than providing clear information.

Whether or not the label is used, it is essential to discuss the symptoms that are likely to prove most difficult when the patient is at home. In about a quarter of cases symptoms do not recur, but it would be a bold clinician who would be absolutely confident that a particular patient would be one of the fortunate ones. The question for the psychiatrist must be-have the relatives all the information they need to cope as effectively as possible with any exacerbation of symptoms, with the side-effects of medication and with any continuing disability?

The longer the disorder persists, the more important it is for there to be a frank discussion of the implications of the term 'schizophrenia'.

\section{Health education}

In the days when patients who were most severely disturbed and chronically disabled were more than likely to become long-term residents of mental hospitals, relatives were relieved of the day-to-day responsibility of living with schizophrenia. Today, without the benefit of consultation, relatives are being asked to provide, in respect of one particular individual, the same sorts of care that psychiatric nurses gave. Indeed, they are the true 'primary care' givers. They often accept this responsibility for years on end, without benefit of shift relief or holidays and with little thanks by way of statutory recognition. The very least that professional care workers can do to ease the burden is to ensure that they are brought into the process of long-term care, in the same way that they would be if they were helping a physically disabled relative.

The appropriate group to help professional bodies, such as the Royal College of Psychiatrists, to draw up a statement of the principles of health education covering these problems would be the National Schizophrenia Fellowship. Their paper on 'Good Relations', mentioned in the Bulletin (August 1984, 8, 161), is a sound and sensible document and others are available ${ }^{1}$ that are eminently suitable for psychiatrists to hand to relatives. There is a useful literature on which to build. The advantages of collaboration, both with a national organization of this kind and with individual relatives throughout the country, 
would be substantial. Relatives would gain greater understanding of schizophrenia and thus be better able to help patients acquire self-knowledge. Greater independence and fewer relapses should result. Professional carers would find their work more rewarding: 'community care' would become less of a slogan and more of a true interaction of benefit to those who badly need help.

The principles of specific (as opposed to general) health education cannot be laid down as systematically or precisely as they can for conditions such as diabetes or even dementia. But we now have sufficient knowledge to ensure that caring relatives who wish for, and can cope with, such help can become reasonably well-informed. To make this information readily available should be one of the major tasks of psychiatrists and of the College.

REFERENCE

'RoLLIN, H. (ed) (1980) Coping with Schizophrenia: The National Schizophrenia Fellowship. London: Burnett Books and Andre Deutsch.

\section{Limiting the Range of Drugs Prescribable under the NHS}

After the meeting of Council on 15 February 1985, the President sent the following comments on the Government's proposals to Mr Norman Fowler, Secretary of State for Social Services.

(1) The College is in favour of rationalizing the prescribing of drugs and ensuring the best use of finite resources, but believes that there are better ways of achieving the desired results. We have serious reservations about the effect of the proposals on the care of our patients and we wish to object to the unrealistically short period of consultation offered.

(2) The College is opposed to a statutory 'restricted list' As well as being unacceptable in principle, it is anticipated that such a list would require cumbersome and continuous revision and a complicated appeals machinery. Paradoxical situations can be envisaged in which special arrangements would have to be made for private prescriptions to be dispensed for NHS hospital in-patients.

(3) The College is opposed to proposals that limit the drugs available in NHS practice while allowing a wider range in private practice. Doctors will be faced with a difficult ethical conflict between prescribing the best treatment available for patients and prescribing the treatment the patient can afford.

(4) The proposals put forward are unacceptably rigid in view of the range of widely differing clinical conditions that are treated with one compound and of the complex and varied nature of compounds that may be needed to treat a single clinical condition. For instance, in the case of benzodiazepines, what applies to the treatment of a neurosis does not necessarily apply to treatment of epilepsy or spastic paraplegia, nor is appropriate to their use in intubation procedures. Benzodiazepines vary widely in their profile of action, their formulation and aspects of pharmacokinetics. Moreover, the pharmacokinetics of drugs varies in sometimes unpredictable ways, e.g. in the elderly. The suggestion that the proposals affect only minor and self-limiting complaints is unfortunate. Constipation in elderly patients can be a major clinical problem. A wide range of laxatives is also required as part of the treatment of more serious conditions (for example, constipation caused by opiates in patients with terminal cancer).

(5) The present proposals carry the inherent and unacceptable risk that more dangerous drugs might be prescribed in place of the restricted drugs (e.g. barbiturates or neuroleptics instead of benzodiazepines or opiates instead of aspirin).

(6) The College is concerned that this proposal will not only cause extra expenditure for sick and needy patients, but that some patient groups, whose prescriptions are free at present, would have to pay the full cost of the drug in future.

\section{'Speculations in Science and Technology'}

Speculations in Science and Technology publishes speculative papers in the physical, mathematical, biological and engineering sciences, together with contributions in interdisciplinary areas. It is published five times a year.
Submissions are now invited and should be sent to the Editor, Dr Alan L. Mackay, Department of Crystallography, Birkbeck College, Malet Street, London WC1E 7HX. 